\title{
Implementation of the Finite Element Technique to the Optimal Control of One Dimensional Energized Wave Equation
}

\author{
BAWA, Musa \\ Department of Mathematics/ Computer Science, Ibrahim Badamasi Babangida University, Lapai, Nigeria.
}

\begin{abstract}
This research computes the optimal control and state of the one-dimentional energized wave equation using the Finite Element Technique ( FET). The paper has to do with all the vital computational elements as in the derivation of the finite element algorithm. With these recalls, various numerical optimal controls and states were considered at various levels of discretization.
\end{abstract}

Keywords: Differential Equation, Energized Wave Equation, Finite Element Technique, Optimal Control, Optimal State.

\section{Introduction}

In theoretical physics and engineering, partial differential equations generally arise from the mathematical formulation of real life physical problems as in Raisinghania [1]. The applications of optimization methods to equations in mathematical physics have been considered by Reju etal [2]. They apply the extended conjugate gradient method to the control problems of diffusion, fluid dynamics and wave propagation. However, there are other methods applied to optimal control of wave propagation with energy effect. In this research work, the Finite Element Technique is used to solve the optimal control problem of wave equation with energy effects in one dimension following the algorithms in Bawa[3].

\section{One Dimensional Energized Wave Equation}

Cases of manageable energy losses can be modeled as a combination of the wave and energy equations simply, this is considered as wave equation with energy effect or energized wave equation. According to pain [4], the one-dimensional wave equation with energy effect is given by:

$$
\frac{\partial^{2} \mathrm{z}(\mathrm{x}, \mathrm{t})}{\partial \mathrm{x}^{2}}=\frac{1}{\mathrm{C}^{2}} \frac{\partial^{2} \mathrm{z}(\mathrm{x}, \mathrm{t})}{\partial \mathrm{t}^{2}}+\frac{1}{\mathrm{~d}} \frac{\partial \mathrm{z}(\mathrm{x}, \mathrm{t})}{\partial \mathrm{t}}
$$

Where $\mathrm{C}$ and $\mathrm{d}$ are the material property Constants namely the wave velocity and energy respectively. The wave part is:

$$
\frac{\partial^{2} \mathrm{z}(\mathrm{x}, \mathrm{t})}{\partial \mathrm{x}^{2}}=\frac{1}{\mathrm{C}^{2}} \frac{\partial^{2} \mathrm{z}(\mathrm{x}, \mathrm{t})}{\partial \mathrm{t}^{2}}
$$

And the energy part is:

$$
\frac{\partial^{2} \mathrm{z}(\mathrm{x}, \mathrm{t})}{\partial \mathrm{x}^{2}}=\frac{1}{\mathrm{~d}} \frac{\partial \mathrm{z}(\mathrm{x}, \mathrm{t})}{\partial \mathrm{t}}
$$

Our optimization problem under consideration is:

$$
\operatorname{Min} \int[\mathrm{z}, \mathrm{u}]=\operatorname{Min} \int_{0}^{1} \int_{0}^{1}\left\{u^{2}(x, t)+z^{2}(x, t)\right\} \mathrm{dxdt}
$$

Subject to:

$$
\frac{\partial^{2} \mathrm{z}(\mathrm{x}, \mathrm{t})}{\partial \mathrm{x}^{2}}+\frac{\partial \mathrm{z}(\mathrm{x}, \mathrm{t})}{\partial \mathrm{t}}=\frac{\partial^{2} \mathrm{z}(\mathrm{x}, \mathrm{t})}{\partial \mathrm{x}^{2}}+\mathrm{u}(\mathrm{x}, \mathrm{t})
$$

With boundary and initial conditions:

$$
\begin{aligned}
& \mathrm{Z}(0, \mathrm{t})=\mathrm{Z}(1, \mathrm{t})=0 ; 0 \leq \mathrm{t} \leq 1 \\
& \mathrm{Z}(\mathrm{x}, 0)=\mathrm{Z}_{\mathrm{o}}(\mathrm{x}) ; \quad 0 \leq \mathrm{x} \leq 1
\end{aligned}
$$

Where $\mathrm{u}(\mathrm{x}, \mathrm{t})$ is the Control or input function.

Writing the Hamiltonian for (2.4) and (2.5), similar to that of Singh and Tiltli [5], we have: 
$\mathrm{H}=\mathrm{Z}^{2}(\mathrm{x}, \mathrm{t})+\mathrm{U}^{2}(\mathrm{x}, \mathrm{t})+\lambda^{\mathrm{T}}\left[\frac{\partial^{2} \mathrm{z}(\mathrm{x}, \mathrm{t})}{\partial \mathrm{x}^{2}}+\mathrm{u}(\mathrm{x}, \mathrm{t})\right]$

Setting:

$$
\text { Where } \lambda^{T}=\lambda^{T}(t)
$$

And:

$$
F[z(x, t), u(x, t)]=\frac{\partial^{2} z(x, t)}{\partial x^{2}}+u(x, t)
$$
optimization as:

$\mathrm{g}\left[(\mathrm{z}(\mathrm{x}, \mathrm{t}), \mathrm{u}(\mathrm{x}, \mathrm{t})]=\mathrm{z}^{2}(\mathrm{x}, \mathrm{t})+\mathrm{u}^{2}(\mathrm{x}, \mathrm{t})\right.$, Consequently, we have the first order necessary conditions for

$$
\begin{aligned}
& \frac{\partial \mathrm{z}(\mathrm{x}, \mathrm{t})}{\partial \mathrm{t}}=\frac{\partial \mathrm{H}(\mathrm{x}, \mathrm{t})}{\partial \lambda}=\frac{\partial^{2} \mathrm{z}(\mathrm{x}, \mathrm{t})}{\partial \mathrm{x}^{2}}+\mathrm{u}(\mathrm{x}, \mathrm{t})=\mathrm{F}[\mathrm{z}(\mathrm{x}, \mathrm{t}), \mathrm{u}(\mathrm{x}, \mathrm{t})] \\
& \frac{\partial \lambda}{\partial \mathrm{t}}=-\frac{\partial \mathrm{H}}{\partial \mathrm{Z}}=-\left[\frac{\partial f}{\partial \mathrm{z}}\right]^{T}-\frac{\partial \mathrm{g}}{\partial \mathrm{z}}=-2 \mathrm{z}(\mathrm{x}, \mathrm{t}) \\
& \frac{\partial \mathrm{H}}{\partial \mathrm{u}}=0 \\
& \begin{array}{ll}
\text { Or } \quad\left[\frac{\partial \mathrm{f}}{\partial \mathrm{u}}\right] \underset{\lambda^{\mathrm{T}}}{\mathrm{T}} \frac{\partial \mathrm{g}}{\partial \mathrm{u}}=0
\end{array}
\end{aligned}
$$

Where:

$$
H=g(z, u)+\lambda^{T}(t) f(z, u)
$$

Equation (2.9) gives:

$\lambda+2 \mathrm{u}(\mathrm{x}, \mathrm{t})=0$

or $\lambda=-2 \mathrm{u}(\mathrm{x}, \mathrm{t})$

using equations (2.8) and (2.10), gives:

$$
\frac{\partial \lambda}{\partial \mathrm{t}}=\frac{2 \partial \mathrm{u}(\mathrm{t}, \mathrm{x})}{\partial \mathrm{t}}=-2 \mathrm{z}(\mathrm{x}, \mathrm{t}),
$$

Which implies that:

$$
\mathrm{Z}(\mathrm{x}, \mathrm{t})=-\frac{\partial \mathrm{u}(\mathrm{t}, \mathrm{x})}{\partial \mathrm{t}}
$$

Equation (2.11) is here of physical significance under the conditions for optimality and it expresses the relationship between the temperature and the heat source at any point $\mathrm{x}$ of the unit propagating rod of our diffused model. Moreover, (2.11) is in this case treated as a differential transform of any previous known solution of the wave diffusion equation.

Assuming that (2.11) admits the fourier solutions proposed by Duchateau and zachmann [6]:.

$$
\begin{aligned}
& \mathrm{Z}(\mathrm{x}, \mathrm{t})=\sum_{i=1}^{\infty} \propto_{1}(\mathrm{t}) \sin \pi \mathrm{ix} \\
& \mathrm{u}(\mathrm{x}, \mathrm{t})=\sum_{i=1}^{\infty} \mathrm{U}_{\mathrm{i}}(\mathrm{t}) \sin \pi \mathrm{ix}
\end{aligned}
$$

We then have our new solution as:

$$
\begin{aligned}
& Z(x, t)=\frac{\partial}{\partial t}\left[\sum_{i=1}^{\infty} U_{i}(t) \sin \pi i x\right] \\
& =\sum_{i=1}^{\infty} U_{i t}(t) \sin \pi i x
\end{aligned}
$$

It follows that:

And:

$$
\propto_{1}(\mathrm{t})=U_{i t}(t)
$$

$$
\begin{gathered}
Z_{t}(x, t)=\sum_{i=1}^{\infty} U_{i t t}(t) \sin \pi i x \\
Z_{t t}(x, t)=\sum_{i=1}^{\infty} U_{i t t t}(t) \sin \pi i x \\
Z_{x x}(x, t)=\sum_{i=1}^{\infty} i^{2}\left(-\pi^{2}\right) U_{i t}(t) \sin \pi i x
\end{gathered}
$$


From our constrained equations:

$$
Z(x, 0)=\sum_{i=1}^{\infty} U_{i t}(0) \sin \pi i x
$$

This implies that:

$$
Z_{t t}(x, t)+Z_{t}(x, t)=Z_{x x}(x, t)+U(x, t)
$$

Thus we have:

$$
\begin{gathered}
\sum_{i=1}^{\infty} U_{t t t}(t) \sin \pi i x+\sum_{i=1}^{\infty} U_{t t}(t) \sin \pi i x \\
=\sum_{i=1}^{\infty} i^{2}\left(-\pi^{2}\right) U_{t t}(t) \sin \pi i x+\sum_{i=1}^{\infty} U_{i}(t) \sin \pi i x
\end{gathered}
$$

$$
U_{i t t t}=U_{i t t}-i^{2} \pi^{2} U_{i t}+U_{i}
$$

Now, the problem can be put in the form:

$$
\begin{aligned}
& \operatorname{Min} \int_{0}^{1}\left[U_{1}+U_{2}+\ldots+U_{n}\right] d t+\int_{0}^{1}\left[U_{1 t}+U_{2 t}+\ldots+U_{n t}\right] d t+ \\
& \int_{0}^{1}\left[U_{1 t t}+U_{2 t t}+\ldots+U_{n t t}\right] d t
\end{aligned}
$$

Subject to the set of equations below:

$$
\begin{gathered}
U_{1 t t t}=-U_{1 t t}-\pi^{2} 1^{2} U_{1 t}+U_{1} \\
U_{2 t t t}=-U_{2 t t}-\pi^{2} 2^{2} U_{2 t}+U_{2} \\
\dot{\cdot} \\
\dot{\cdot} \\
\dot{U_{n t t t}}=-U_{n t t}-\pi^{2} n^{2} U_{n t}+U_{n}
\end{gathered}
$$

\section{The Discretization and Interpolation Model .}

Solving system (2.16) using the finite element technique as applied in Rao [7], we discretize the domain ( $t=0$ to 1 ) with elements of equal length. For the interpolation model, we consider a one-dimensional ( line segment) of length $\mathrm{L}$ with two nodes, one at each end. Let the nodes be denoted by $\mathrm{i}$ and $\mathrm{j}$ and the nodal values of the field variable $U$ by $U_{i}$ and $U_{j}$

The variation of $\mathrm{U}$ inside the element is assumed to be linear as:

$$
U(t)=\propto_{1}+\propto_{2} t
$$

Where $\propto_{1}$ and $\propto_{2}$ are the unknown coefficients.

Using the nodal conditions:

From equation (3.1):

$$
\begin{gathered}
U(t)=U_{i} \text { at } t=t_{i} \\
U(t)=U_{j} \text { at } t=t_{j}
\end{gathered}
$$

$$
\begin{aligned}
& U_{i}=\propto_{1}+\propto_{2} t_{i} \\
& U_{j}=\propto_{1}+\propto_{2} t_{j}
\end{aligned}
$$

Solving equations (3.2) and (3.3) gives:

$$
\propto_{1}=\frac{U_{i} t_{j}-U_{j} t_{i}}{L}
$$




$$
\propto_{2}=\frac{U_{j}-U_{i}}{L}
$$

$\mathrm{L}=t_{j}-t_{i}$

Where $t_{i}$ and $t_{j}$ denote the global co-ordinates of nodes $\mathrm{i}$ and $\mathrm{j}$ respectively.

Substituting (3.4) in (3.1) gives:

$$
\begin{aligned}
U(t) & =N_{i}(t) U_{i}+N_{j}(t) U_{j} \\
& =[N(t)] U^{(e)}
\end{aligned}
$$

Where:

$$
N_{i}(t)=\left(\frac{t_{j}-t}{L}\right) \quad N_{j}(t)=\left(\frac{t-t_{i}}{L}\right)
$$

$U^{(e)}=\left\{\begin{array}{l}U_{i} \\ U_{j}\end{array}\right\}$ Vector of nodal unknowns of element $\mathrm{e}$

\section{Element Characteristics Matrices and Vectors}

Equation (2.16) is the same as:

$$
\frac{\partial^{3} U_{n}}{\partial t^{3}}=\frac{\partial^{2} U_{n}}{\partial t^{2}}-\pi^{2} n^{2} \frac{\partial U_{n}}{\partial t}+U_{n}
$$

The necessary Conditions for optimality lead to the functional:

Minimize I $=\frac{1}{2} \int\left[-\left[\frac{\partial^{2} u_{n}}{\partial t^{2}}\right]^{2}+\left[\frac{\partial U_{n}}{\partial t}\right]^{2}+2 \pi^{2} n^{2} U_{n}-U n^{2}\right] d t$

The element characteristic matrices and vectors can be identified by expressing the functional I in matrix form. We express the functional I as a sum of $\mathrm{E}$ elemental quantities $I^{(e)}$ as

\section{Where:}

$$
I=\sum_{e=1}^{E} 1^{(e)}
$$

$I^{(e)}=\frac{1}{2} \int_{t_{i}}^{t_{j}}\left[-\left[\frac{\partial^{2} u_{n}}{\partial t^{2}}\right]^{2}+\left[\frac{\partial U_{n}}{\partial t}\right]^{2}+2 \pi^{2} n^{2} U_{n}-U n^{2}\right] d t$

Substituting (3.5) in (4.2) gives:

$I^{(e)}=$

$\frac{1}{2} \int_{t_{i}}^{t_{j}}\left[-U_{n} \rightarrow(e)^{T}\left[\frac{\partial^{2} N}{\partial t^{2}}\right]^{T}\left[\frac{\partial^{2} N}{\partial t^{2}}\right] U_{n}{ }^{\rightarrow(e)}+U_{n} \rightarrow(e)^{T}\left[\frac{\partial N}{\partial t}\right]^{T}\left[\frac{\partial N}{\partial t}\right] U_{n} \rightarrow^{(e)}+2 \pi^{2} n^{2}[N] U_{n} \rightarrow(e)-\right.$

UneTNTN Un $\rightarrow$ edt

For the stationeries of I, we use the necessary conditions:

$$
\frac{\partial I}{\partial U_{i}}=\sum_{e=1}^{E} \frac{\partial I^{(e)}}{\partial U_{i}}=0
$$

$\mathrm{i}=1,2 \ldots \mathrm{M}$

Where $\mathrm{E}$ is the number of elements and $\mathrm{M}$ is the number of nodal degrees of freedom. Equation (4.4) can also be expressed as:

That is:

$$
\sum_{e=1}^{E} \frac{\partial I^{(e)}}{\partial U^{\rightarrow(e)}}=0^{\rightarrow}
$$




$$
\sum_{e=1}^{E} \int_{t_{i}}^{t_{j}}\left[\begin{array}{c}
{\left[\frac{\partial^{2} N}{\partial t^{2}}\right]^{T}\left[\frac{\partial^{2} N}{\partial t^{2}}\right] U_{n}^{\rightarrow(e)}+\left[\frac{\partial N}{\partial t}\right]^{T}\left[\frac{\partial N}{\partial t}\right] U_{n}{ }^{(e)}+\pi^{2} n^{2}[N]^{T}} \\
-[N]^{T}[N] U_{n}{ }^{(e)}
\end{array}\right] d t=0
$$

This implies that:

$$
\sum_{e=1}^{E}\left[k^{(e)}\right] U_{n} \rightarrow(e)=\sum_{e=1}^{E} P^{\rightarrow}(e)
$$

Where $K^{(e)}$ is the element characteristic matrix and $P^{\rightarrow(e)}$ is the element characteristic vector.

\section{Assemblage of Element characteristic matrices and vectors.}

We assemble the element characteristic matrices and vectors and obtain the overall equations as:

$$
[K] U_{n} \rightarrow=P^{\rightarrow}
$$

Case $\mathrm{E}=2$

Case $\mathrm{E}=3$

$$
\frac{1}{2}\left[\begin{array}{ccc}
-22 & 25 & 0 \\
25 & -44 & 25 \\
0 & 25 & -22
\end{array}\right]\left[\begin{array}{l}
U_{n_{1}} \\
U_{n_{2}} \\
U_{n_{3}}
\end{array}\right]=\frac{\pi^{2} n^{2}}{4}\left[\begin{array}{l}
1 \\
2 \\
1
\end{array}\right]
$$

Case $\mathrm{E}=4$

$$
\frac{1}{18}\left[\begin{array}{cccc}
-52 & 55 & 0 & 0 \\
55 & -104 & 55 & 0 \\
0 & 55 & -104 & 55 \\
0 & 0 & 55 & -52
\end{array}\right]\left[\begin{array}{l}
U_{n_{1}} \\
U_{n_{2}} \\
U_{n_{3}} \\
U_{n_{4}}
\end{array}\right]=\frac{\pi^{2} n^{2}}{6}\left[\begin{array}{l}
1 \\
2 \\
2 \\
1
\end{array}\right]
$$

$$
\frac{1}{24}\left[\begin{array}{ccccc}
-94 & 97 & 0 & 0 & 0 \\
97 & -188 & 97 & 0 & 0 \\
0 & 97 & -188 & 97 & 0 \\
0 & 0 & 97 & -188 & 97 \\
0 & 0 & 0 & 97 & -94
\end{array}\right]\left[\begin{array}{l}
U_{n_{1}} \\
U_{n_{2}} \\
U_{n_{3}} \\
U_{n_{4}} \\
U_{n_{5}}
\end{array}\right]=\frac{\pi^{2} n^{2}}{8}\left[\begin{array}{l}
1 \\
2 \\
2 \\
2 \\
1
\end{array}\right]
$$

Case $\mathrm{E}=5$

$$
\frac{1}{30}\left[\begin{array}{cccccc}
-148 & 151 & 0 & 0 & 0 & 0 \\
151 & -296 & 151 & 0 & 0 & 0 \\
0 & 151 & -296 & 151 & 0 & 0 \\
0 & 0 & 151 & -296 & 151 & 0 \\
0 & 0 & 0 & 151 & -296 & 151 \\
0 & 0 & 0 & 0 & 151 & -148
\end{array}\right]\left[\begin{array}{l}
U_{n_{1}} \\
U_{n_{2}} \\
U_{n_{3}} \\
U_{n_{4}} \\
U_{n_{5}} \\
U_{n_{6}}
\end{array}\right]=\frac{\pi^{2} n^{2}}{10}\left[\begin{array}{l}
1 \\
2 \\
2 \\
2 \\
2 \\
1
\end{array}\right]
$$

'Case $\mathrm{E}=6$

$$
\frac{1}{36}\left[\begin{array}{ccccccc}
-214 & 217 & 0 & 0 & 0 & 0 & 0 \\
217 & -428 & 217 & 0 & 0 & 0 & 0 \\
0 & 217 & -428 & 217 & 0 & 0 & 0 \\
0 & 0 & 217 & -428 & 0 & 0 & 0 \\
0 & 0 & 0 & 217 & 217 & 217 & 0 \\
0 & 0 & 0 & 0 & -428-428 & 217 \\
0 & 0 & 0 & 0 & 217 & 217 & -428
\end{array}\right]\left[\begin{array}{l}
U_{n_{1}} \\
U_{n_{2}} \\
U_{n_{3}} \\
U_{n_{4}} \\
U_{n_{5}} \\
U_{n_{6}} \\
U_{n_{7}}
\end{array}\right]=\frac{\pi^{2} n^{2}}{12}\left[\begin{array}{c}
1 \\
2 \\
2 \\
2 \\
2 \\
2 \\
1
\end{array}\right]
$$


Case $\mathrm{E}=7$

$$
\frac{1}{42}\left[\begin{array}{cccccccc}
-292 & 295 & 0 & 0 & 0 & 0 & 0 & 0 \\
295 & -584 & 295 & 0 & 0 & 0 & 0 & 0 \\
0 & 295 & -584 & 295 & 0 & 0 & 0 & 0 \\
0 & 0 & 295 & -584 & 295 & 0 & 0 & 0 \\
0 & 0 & 0 & 295 & -584 & 295 & 0 & 0 \\
0 & 0 & 0 & 0 & 295 & -584 & 295 & 0 \\
0 & 0 & 0 & 0 & 0 & 295 & -584 & 295 \\
0 & 0 & 0 & 0 & 0 & 0 & 295 & -292
\end{array}\right]\left[\begin{array}{l}
U_{n_{1}} \\
U_{n_{2}} \\
U_{n_{3}} \\
U_{n_{4}} \\
U_{n_{5}} \\
U_{n_{6}} \\
U_{n_{7}} \\
U_{n_{8}}
\end{array}\right]=\frac{\pi^{2} n^{2}}{14}\left[\begin{array}{c}
1 \\
2 \\
2 \\
2 \\
2 \\
2 \\
2 \\
1
\end{array}\right]
$$

Case $\mathrm{E}=8$

$$
\frac{1}{48}\left[\begin{array}{ccccccccc}
-382 & 385 & 0 & 0 & 0 & 0 & 0 & 0 & 0 \\
385 & -764 & 385 & 0 & 0 & 0 & 0 & 0 & 0 \\
0 & 385 & -764 & 385 & 0 & 0 & 0 & 0 & 0 \\
0 & 0 & 385 & -764 & 385 & 0 & 0 & 0 & 0 \\
0 & 0 & 0 & 385 & -764 & 385 & 0 & 0 & 0 \\
0 & 0 & 0 & 0 & 385 & -764 & 385 & 0 & 0 \\
0 & 0 & 0 & 0 & 0 & 385 & -764 & 385 & 0 \\
0 & 0 & 0 & 0 & 0 & 0 & 385 & -764 & 385 \\
0 & 0 & 0 & 0 & 0 & 0 & 0 & 385 & -382
\end{array}\right]\left[\begin{array}{l}
U_{n_{1}} \\
U_{n_{2}} \\
U_{n_{3}} \\
U_{n_{4}} \\
U_{n_{5}} \\
U_{n_{6}} \\
U_{n_{7}} \\
U_{n_{8}} \\
U n_{9}
\end{array}\right]=\frac{\pi^{2} n^{2}}{16}\left[\begin{array}{c}
1 \\
2 \\
2 \\
2 \\
2 \\
2 \\
2 \\
2 \\
1
\end{array}\right]
$$

Case $\mathrm{E}=9$

$$
\frac{1}{54}\left[\begin{array}{cccccccccc}
-484 & 487 & 0 & 0 & 0 & 0 & 0 & 0 & 0 & 0 \\
487 & -968 & 487 & 0 & 0 & 0 & 0 & 0 & 0 & 0 \\
0 & 487 & -968 & 487 & 0 & 0 & 0 & 0 & 0 & 0 \\
0 & 0 & 487 & -968 & 487 & 0 & 0 & 0 & 0 & 0 \\
0 & 0 & 0 & 487 & -968 & 487 & 0 & 0 & 0 & 0 \\
0 & 0 & 0 & 0 & 487 & -968 & 487 & 0 & 0 & 0 \\
0 & 0 & 0 & 0 & 0 & 487 & -968 & 487 & 0 & 0 \\
0 & 0 & 0 & 0 & 0 & 0 & 487 & -968 & 487 & 0 \\
0 & 0 & 0 & 0 & 0 & 0 & 0 & 487 & -968 & 487 \\
0 & 0 & 0 & 0 & 0 & 0 & 0 & 0 & 487 & -484
\end{array}\right]\left[\begin{array}{c}
U_{n_{1}} \\
U_{n_{2}} \\
U_{n_{3}} \\
U_{n_{4}} \\
U_{n_{5}} \\
U_{n_{6}} \\
U_{n_{7}} \\
U_{n_{8}} \\
U_{n_{9}} \\
U_{n_{10}}
\end{array}\right]=\frac{\pi^{2} n^{2}}{18}\left[\begin{array}{c}
1 \\
2 \\
2 \\
2 \\
2 \\
2 \\
2 \\
2 \\
2 \\
1
\end{array}\right]
$$

Case $\mathrm{E}=10$

$$
\frac{1}{60}\left[\begin{array}{ccccccccccc}
-598 & 601 & 0 & 0 & 0 & 0 & 0 & 0 & 0 & 0 & 0 \\
601 & -1196 & 601 & 0 & 0 & 0 & 0 & 0 & 0 & 0 & 0 \\
0 & 601 & -1196 & 601 & 0 & 0 & 0 & 0 & 0 & 0 & 0 \\
0 & 0 & 601 & -1196 & 601 & 0 & 0 & 0 & 0 & 0 & 0 \\
0 & 0 & 0 & 601 & -1196 & 601 & 0 & 0 & 0 & 0 & 0 \\
0 & 0 & 0 & 0 & 601 & -1196 & 601 & 0 & 0 & 0 & 0 \\
0 & 0 & 0 & 0 & 0 & 601 & -1196 & 601 & 0 & 0 & 0 \\
0 & 0 & 0 & 0 & 0 & 0 & 601 & -1196 & 601 & 0 & 0 \\
0 & 0 & 0 & 0 & 0 & 0 & 0 & 601 & -1196 & 601 & 0 \\
0 & 0 & 0 & 0 & 0 & 0 & 0 & 0 & 601 & -1196 & 601 \\
0 & 0 & 0 & 0 & 0 & 0 & 0 & 0 & 0 & 601 & -598
\end{array}\right]\left[\begin{array}{c}
U_{n_{1}} \\
U_{n_{2}} \\
U_{n_{3}} \\
U_{n_{4}} \\
U_{n_{5}} \\
U_{n_{6}} \\
U_{n_{7}} \\
U_{n_{8}} \\
U_{n_{9}} \\
U_{n_{10}} \\
U_{n_{11}}
\end{array}\right]=\frac{\pi^{2} n^{2}}{20}\left[\begin{array}{c}
1 \\
2 \\
2 \\
2 \\
2 \\
2 \\
2 \\
2 \\
2 \\
1
\end{array}\right]
$$

\section{Computational Results.}

We can solve the system equations (5.1) after incorporating the boundary conditions:

Case $\mathrm{E}=2$

$$
\left[\begin{array}{l}
U_{n_{1}} \\
U_{n_{2}} \\
U_{n_{3}}
\end{array}\right]=\left[\begin{array}{c}
0 \\
-0.1363636 \pi^{2} n^{2} \\
0
\end{array}\right]\left[\begin{array}{l}
Z_{n_{1}} \\
Z_{n_{2}} \\
Z_{n_{3}}
\end{array}\right]=\left[\begin{array}{c}
0 \\
0.2727273 \pi^{2} n^{2} \\
0
\end{array}\right]
$$


Case $\mathrm{E}=3$

$$
\left[\begin{array}{l}
U_{n_{1}} \\
U_{n_{2}} \\
U_{n_{3}} \\
U_{n_{4}}
\end{array}\right]=\left[\begin{array}{c}
0 \\
-0.122449 \pi^{2} n^{2} \\
-0.122449 \pi^{2} n^{2} \\
0
\end{array}\right]\left[\begin{array}{l}
Z_{n_{1}} \\
Z_{n_{2}} \\
Z_{n_{3}} \\
Z_{n_{4}}
\end{array}\right]=\left[\begin{array}{c}
0 \\
0.367347 \pi^{2} \\
0 \\
0
\end{array}\right]
$$

Case $\mathrm{E}=4$

$$
\left[\begin{array}{l}
U_{n_{1}} \\
U_{n_{2}} \\
U_{n_{3}} \\
U_{n_{4}} \\
U_{n_{5}}
\end{array}\right]=\left[\begin{array}{c}
0 \\
-0.1034733 \pi^{2} n^{2} \\
-0.1386905 \pi^{2} n^{2} \\
-0.1034733 \pi^{2} n^{2} \\
0
\end{array}\right]\left[\begin{array}{l}
Z_{n_{1}} \\
Z_{n_{2}} \\
Z_{n_{3}} \\
Z_{n_{4}} \\
Z_{n_{5}}
\end{array}\right]=\left[\begin{array}{c}
0 \\
0.4138932 \pi^{2} n^{2} \\
0.1408688 \pi^{2} n^{2} \\
-0.1408688 \pi^{2} n^{2} \\
0
\end{array}\right]
$$

Case $\mathrm{E}=5$

$$
\left[\begin{array}{l}
U_{n_{1}} \\
U_{n_{2}} \\
U_{n_{3}} \\
U_{n_{4}} \\
U_{n_{5}} \\
U_{n_{6}}
\end{array}\right]=\left[\begin{array}{c}
0 \\
-8.857476 E-02 \pi^{2} n^{2} \\
-0.1333068 \pi^{2} n^{2} \\
-0.1333068 \pi^{2} n^{2} \\
-8.857476 E-02 \pi^{2} n^{2} \\
0
\end{array}\right]\left[\begin{array}{l}
Z_{n_{1}} \\
Z_{n_{2}} \\
Z_{n_{3}} \\
Z_{n_{4}} \\
Z_{n_{5}} \\
Z_{n_{6}}
\end{array}\right]=\left[\begin{array}{c}
0 \\
0.4413738 \pi^{2} n^{2} \\
0.2251602 \pi^{2} n^{2} \\
0 \\
-0.2251602 \pi^{2} n^{2} \\
0
\end{array}\right]
$$

Case $\mathrm{E}=6$

$$
\left[\begin{array}{l}
U_{n_{1}} \\
U_{n_{2}} \\
U_{n_{3}} \\
U_{n_{4}} \\
U_{n_{5}} \\
U_{n_{6}} \\
U_{n_{7}}
\end{array}\right]=\left[\begin{array}{c}
0 \\
-7.65768 E-02 \pi^{2} n^{2} \\
-0.1233866 \pi^{2} n^{2} \\
-0.139135 \pi^{2} n^{2} \\
-0.1233866 \pi^{2} n^{2} \\
-7.657682 E-02 \pi^{2} n^{2} \\
0
\end{array}\right]\left[\begin{array}{l}
Z_{n_{1}} \\
Z_{n_{2}} \\
Z_{n_{3}} \\
Z_{n_{4}} \\
Z_{n_{5}} \\
Z_{n_{6}} \\
Z_{n_{7}}
\end{array}\right]=\left[\begin{array}{c}
0 \\
0.4594609 \pi^{2} n^{2} \\
0.2808586 \pi^{2} n^{2} \\
0.0944904 \pi^{2} n^{2} \\
-0.0944904 \pi^{2} n^{2} \\
-0.2808586 \pi^{2} n^{2} \\
0
\end{array}\right]
$$

Case $\mathrm{E}=7$

Case $\mathrm{E}=8$

$$
\left[\begin{array}{l}
U_{n_{1}} \\
U_{n_{2}} \\
U_{n_{3}} \\
U_{n_{4}} \\
U_{n_{5}} \\
U_{n_{6}} \\
U_{n_{7}} \\
U_{n_{8}}
\end{array}\right]=\left[\begin{array}{c}
0 \\
-6.746464 E-02 \pi^{2} n^{2} \\
-0.1132181 \pi^{2} n^{2} \\
-0.1363299 \pi^{2} n^{2} \\
-0.1363299 \pi^{2} n^{2} \\
-0.1132181 \pi^{2} n^{2} \\
-6.746464 E-02 \pi^{2} n^{2} \\
0
\end{array}\right]\left[\begin{array}{l}
Z_{n_{1}} \\
Z_{n_{2}} \\
Z_{n_{3}} \\
Z_{n_{4}} \\
Z_{n_{5}} \\
Z_{n_{6}} \\
Z_{n_{7}} \\
Z_{n_{8}}
\end{array}\right]=\left[\begin{array}{c}
0 \\
0.4772524 \pi^{2} n^{2} \\
0.3202742 \pi^{2} n^{2} \\
0.1617826 \pi^{2} n^{2} \\
0 \\
-0.1617826 \pi^{2} n^{2} \\
-0.3202742 \pi^{2} n^{2} \\
0
\end{array}\right]
$$

$$
\left[\begin{array}{l}
U_{n_{1}} \\
U_{n_{2}} \\
U_{n_{3}} \\
U_{n_{4}} \\
U_{n_{5}} \\
U_{n_{6}} \\
U_{n_{7}} \\
U_{n_{8}} \\
U_{n_{9}}
\end{array}\right]=\left[\begin{array}{c}
0 \\
-0.0602213 \pi^{2} n^{2} \\
-0.1039197 \pi^{2} n^{2} \\
-0.1304141 \pi^{2} n^{2} \\
-0.1392917 \pi^{2} n^{2} \\
-0.1304141 \pi^{2} n^{2} \\
-0.1039197 \pi^{2} n^{2} \\
-0.0602213 \pi^{2} n^{2} \\
0
\end{array}\right]\left[\begin{array}{c}
Z_{n_{1}} \\
Z_{n_{2}} \\
Z_{n_{3}} \\
Z_{n_{4}} \\
Z_{n_{5}} \\
Z_{n_{6}} \\
Z_{n_{7}} \\
Z_{n_{8}} \\
Z_{n_{9}}
\end{array}\right]=\left[\begin{array}{c}
0 \\
0.4817704 \pi^{2} n^{2} \\
0.3495872 \pi^{2} n^{2} \\
0.2119552 \pi^{2} n^{2} \\
0.0710208 \pi^{2} n^{2} \\
-0.0710208 \pi^{2} n^{2} \\
-0.2119552 \pi^{2} n^{2} \\
-0.3495872 \pi^{2} n^{2} \\
0
\end{array}\right]
$$


Case $\mathrm{E}=9$

$$
\left[\begin{array}{c}
U_{n_{1}} \\
U_{n_{2}} \\
U_{n_{3}} \\
U_{n_{4}} \\
U_{n_{5}} \\
U_{n_{6}} \\
U_{n_{7}} \\
U_{n_{8}} \\
U_{n_{9}} \\
U_{n_{10}}
\end{array}\right]=\left[\begin{array}{c}
0 \\
-5.434729 E-02 \pi^{2} n^{2} \\
-9.570468 E-02 \pi^{2} n^{2} \\
-0.1235627 \pi^{2} n^{2} \\
-0.137578 \pi^{2} n^{2} \\
-0.137578 \pi^{2} n^{2} \\
-0.1235627 \pi^{2} n^{2} \\
-9.570468 E-02 \pi^{2} n^{2} \\
-5.434729 E-02 \pi^{2} n^{2} \\
0
\end{array}\right]\left[\begin{array}{c}
Z_{n_{1}} \\
Z_{n_{2}} \\
Z_{n_{3}} \\
Z_{n_{4}} \\
Z_{n_{5}} \\
Z_{n_{6}} \\
Z_{n_{7}} \\
Z_{n_{8}} \\
Z_{n_{9}} \\
Z_{n_{10}}
\end{array}\right]=\left[\begin{array}{c}
0 \\
0.4891257 \pi^{2} n^{2} \\
0.3722166 \pi^{2} n^{2} \\
0.2507221 \pi^{2} n^{2} \\
0.1261377 \pi^{2} n^{2} \\
0 \\
-0.1261377 \pi^{2} n^{2} \\
-0.2507221 \pi^{2} n^{2} \\
-0.3722166 \pi^{2} n^{2} \\
0
\end{array}\right]
$$

Case $\mathrm{E}=10$

$$
\left[\begin{array}{c}
U_{n_{1}} \\
U_{n_{2}} \\
U_{n_{3}} \\
U_{n_{4}} \\
U_{n_{5}} \\
U_{n_{6}} \\
U_{n_{7}} \\
U_{n_{8}} \\
U_{n_{9}} \\
U_{n_{11}}
\end{array}\right]=\left[\begin{array}{c}
0 \\
-4.949809 E-02 \pi^{2} n^{2} \\
-8.851864 E-02 \pi^{2} n^{2} \\
-0.1166721 \pi^{2} n^{2} \\
-0.1336774 \pi^{2} n^{2} \\
-0.1393648 \pi^{2} n^{2} \\
-0.133774 \pi^{2} n^{2} \\
-0.1166721 \pi^{2} n^{2} \\
-8.851864 E-02 \pi^{2} n^{2} \\
-4.949808 E-02 \pi^{2} n^{2} \\
0
\end{array}\right]\left[\begin{array}{c}
Z_{n_{1}} \\
Z_{n_{2}} \\
Z_{n_{3}} \\
Z_{n_{4}} \\
Z_{n_{5}} \\
Z_{n_{6}} \\
Z_{n_{7}} \\
Z_{n_{8}} \\
Z_{n_{9}} \\
Z_{n_{10}} \\
Z_{n_{11}}
\end{array}\right]=\left[\begin{array}{c}
0 \\
0.494980 \pi^{2} n^{2} \\
0.3902051 \pi^{2} n^{2} \\
0.2815346 \pi^{2} n^{2} \\
0.170053 \pi^{2} n^{2} \\
0.056874 \pi^{2} n^{2} \\
-0.056874 \pi^{2} n^{2} \\
-0.170053 \pi^{2} n^{2} \\
-0.2815346 \pi^{2} n^{2} \\
-0.3902051 \pi^{2} n^{2} \\
0
\end{array}\right]
$$

\section{Conclusion}

The research work finds the expressions for the optimal state $\mathrm{Z}(\mathrm{x}, \mathrm{y})$ and the optimal control $\mathrm{U}(\mathrm{x}, \mathrm{y})$ for the one dimensional wave equation with energy effect. The controls are symmetrical about their minimum values and these values get smaller as the number of elements increases. Two and three elements discretisation maintain only positive states with the latter having the same value at $z_{n_{1}}, z_{n_{2}}$ and $z_{n_{4}}$. No other pair exhibits similar behavior. The analytical solutions would enhance further computational processes using the FET towards the derivation of the optimal controls for states at various spatial planes.

\section{References}

[1] M. D. Raisinghania, Advanced differential equations, S. Chand and Company Ltd, New Delhi, 2010

[2] S. A. Reju, M. A. Ibiejugba, and J. D. Evans, Optimal control of the wave propagation problem with the extended conjugate gradient method, Intern. J. Computer Math. 77(3),2001,425-439.

[3] M.Bawa, Application of finite element technique to the optimal control of wave equation with energy effect, unpublished M.Tech thesis, Federal University of Technology, Minna, Nigeria,2001.

[4] H. J.Pain, The physics of vibrations and waves, $2^{\text {nd }}$ edition, John Wiley and Sons, 1979.

[5] M. G. Singh, and A. Titli, Systems (Decomposition, Optimization and Control), Pergamon Press,1978.

[6] P. Duchateau, and D. W. Zachmann, Partial differential equations, McGraw-Hill Publishing Company,1986.

[7] S. S. Rao, The finite element method in engineering, Pergamon Press, 1989. 